\title{
Process sequences in biomimetic research
}

\author{
T. Speck \& O. Speck \\ Plant Biomechanics Group Freiburg, Botanic Garden, \\ University of Freiburg, Faculty of Biology, Freiburg, Germany, \\ and Competence Networks Biomimetics and BIOKON e.V, Germany
}

\begin{abstract}
The entire developmental process from the biological template to the marketable biomimetic product is characterized by close cooperation between biologists, engineers and other scientists involved in the research project, who may come from branches of mathematics, informatics, physics, chemistry, geology, hydrology or meteorology. The presented methodology of doing biomimetics has proven effective in many R\&D projects. Two principally different approaches can be distinguished as 'bottom-up process' and 'top-down process'. Depending on the problem to be solved, numerous transitions exist between the two procedures.
\end{abstract}

Keywords: biomimetics, top-down process, bottom-up process, technical biology.

\section{Introduction}

Biomimetics is a portmanteau fabricated from the words Biology and mimesis (imitation). Its contents are essentially identical with the term bionics (combined from Biology and Technics). As alternatives to 'bionic' or 'biomimetic', the terms 'biologically inspired' or 'bio-inspired' are sometimes used.

Biomimetics is the realization of technical applications based on insights resulting from fundamental biological research. Biomimetic developments are not direct carry-overs from biology, i.e. never 'blueprints from nature'. Biomimetics has to be thought of as a creative technological implementation. It represents a reinvention inspired by nature. Biomimetic research typically includes several levels of abstraction and modification. Biomimetics is an extremely interdisciplinary research discipline in which experts from various areas, such as biologists, chemists, physicists or engineers cooperate in R\&Dprojects. Depending on the scientific expertise of the individual participants, 
research concentrates more on the biological or more on the technical aspects of biomimetics.

In biomimetic research seven subdivisions can be distinguished, fig. 1. The borders of these subdivisions are flexible, and new focal points for biomimetic research keep appearing in the highly dynamic biomimetic research landscape. Therefore, the proposed classification is constantly expanding and is supplemented by new developments [1].

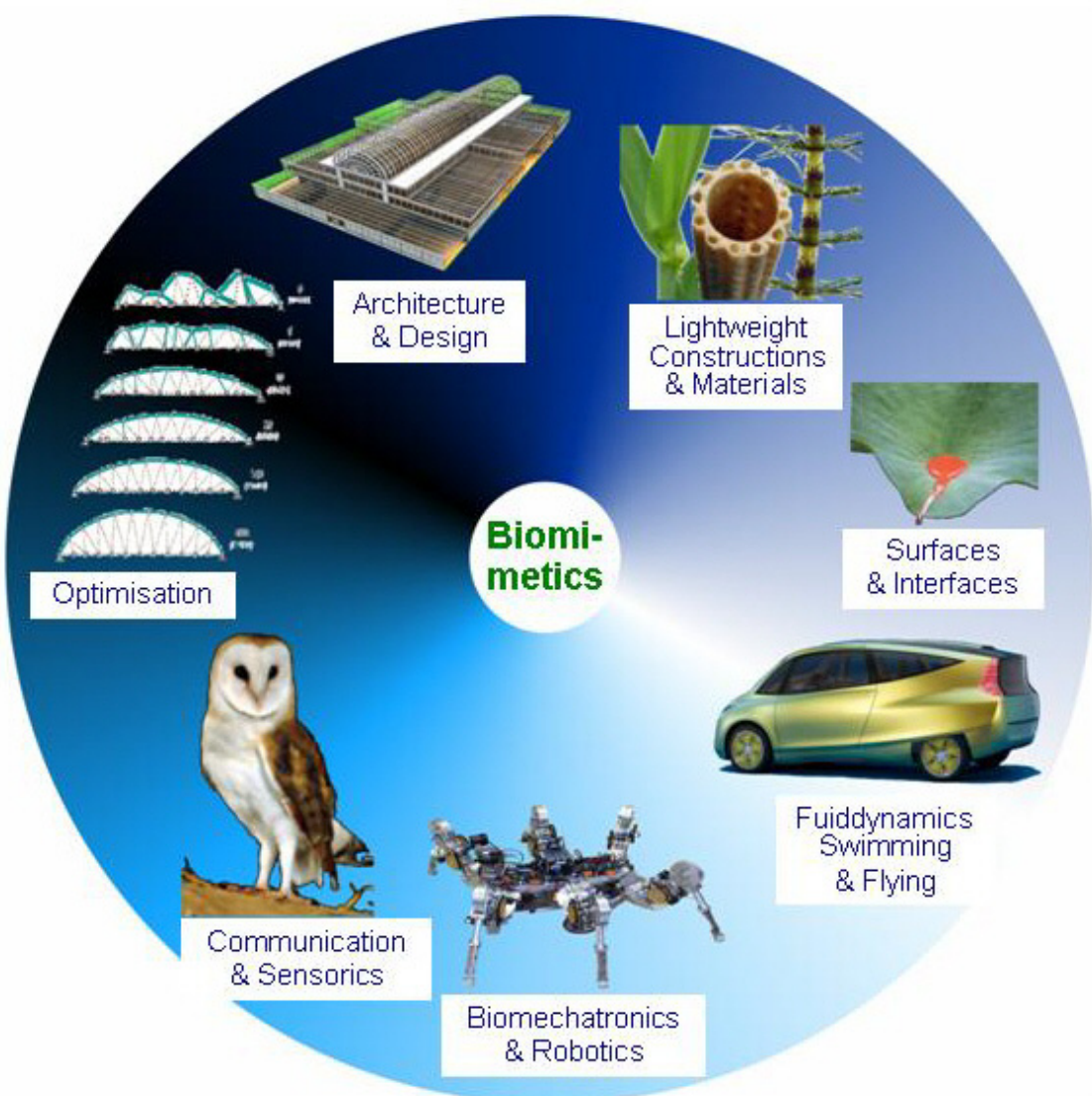

Figure 1: Subdivisions of biomimetics as classified by the Bionics Competence Network BIOKON e.V. (modified from (C) Schrödel Verlag).

The term 'technical biology' was established by Werner Nachtigall as a complementary item to biomimetics (bionics) [2,3]. Technical biology stands for the analysis of form-structure-function-relationships in living organisms using methodological approaches from physics and engineering sciences. Technical biology is the basis of many biomimetic research projects as it allows one to 
understand the functioning of the biological templates in a quantitative and technologically based manner. These quantitative analyses are the basis for abstracting and transferring ideas from biology to technical applications in the course of biomimetic projects, fig 2. Since a couple of years ago it appears that findings during the implementation of functional principles inspired from biology in innovative biomimetic products may also contribute to a better understanding of biological systems. This relatively new insight of a transfer process that can be referred to as 'reverse biomimetics' can be interpreted as closing the heuristic spiral of technical biology, biomimetics and reverse biomimetics.

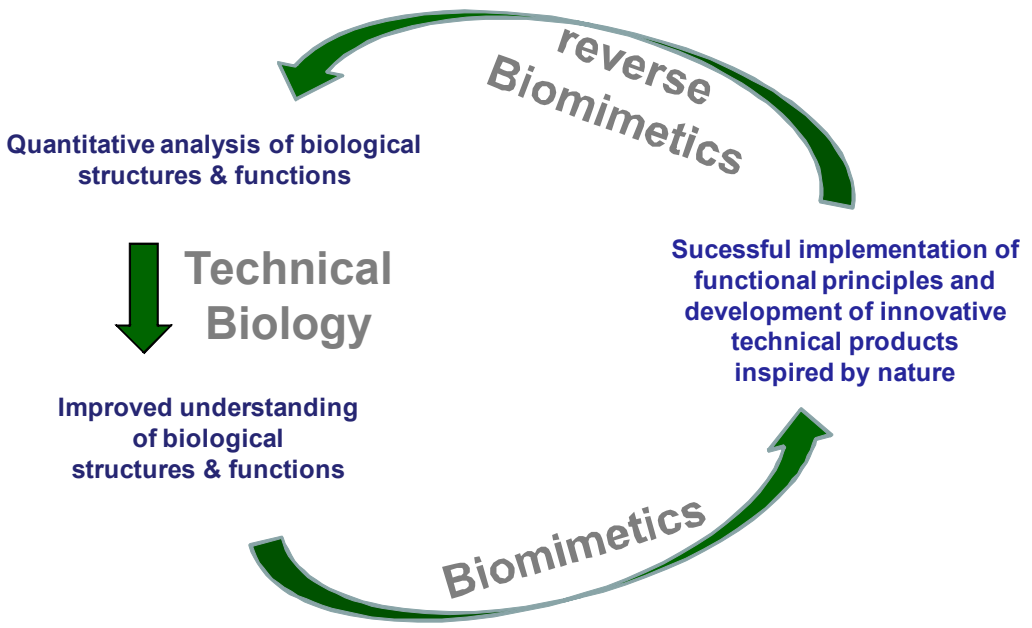

Figure 2: Relation and mode of operation of technical biology and biomimetics.

In this context, it seems useful also to distinguish between biomimetics and biotechnology, which are the two most important areas of translational biology, i.e. of application-oriented biological research.

Biotechnology clearly deals with micro- and molecular biological as well as biochemical contents. By biotechnology we mean the use of (genetically altered) organisms to produce desired substances or to degrade undesirable substances. In biotechnology theses organisms (bacteria, protozoa, fungi, plants, animals) are directly involved in the process of production or degradation.

In biomimetics (bionics), on the other hand, living organisms function as concept generators for innovative technical applications but are not directly involved in the production of biomimetic products.

In most cases, based on these definitions a research project can be classified as belonging either to biomimetics or to biotechnology. However, there exist projects in which the boundary between biotechnology and biomimetics becomes invisible. One such example is the development of synthetic spider silk currently being promoted by several research groups. The production of the base material 
(spider silk protein) is brought about by genetically manipulated bacteria or animals and therefore clearly belongs to the biotechnological area. On the other hand, the applied methodology for spinning out the silk threads by using spinning devices inspired by the spinnerets of spiders has to be assigned to biomimetics.

\section{Bottom-up process}

In this approach new biomimetic research projects for technical implementation are born from new and promising results of fundamental biological research, fig. 3. The first process step (in the presented example) is to analyse the biomechanics and functional morphology of a biological system. In the next step quantitative analysis leads to a principal and detailed understanding of the biological structures, shapes and functions. On the abstraction level, which follows next, separation of the principles discovered from the biological model takes place. Abstraction often proves to be one of the most important as well as most difficult steps in a biomimetic project.

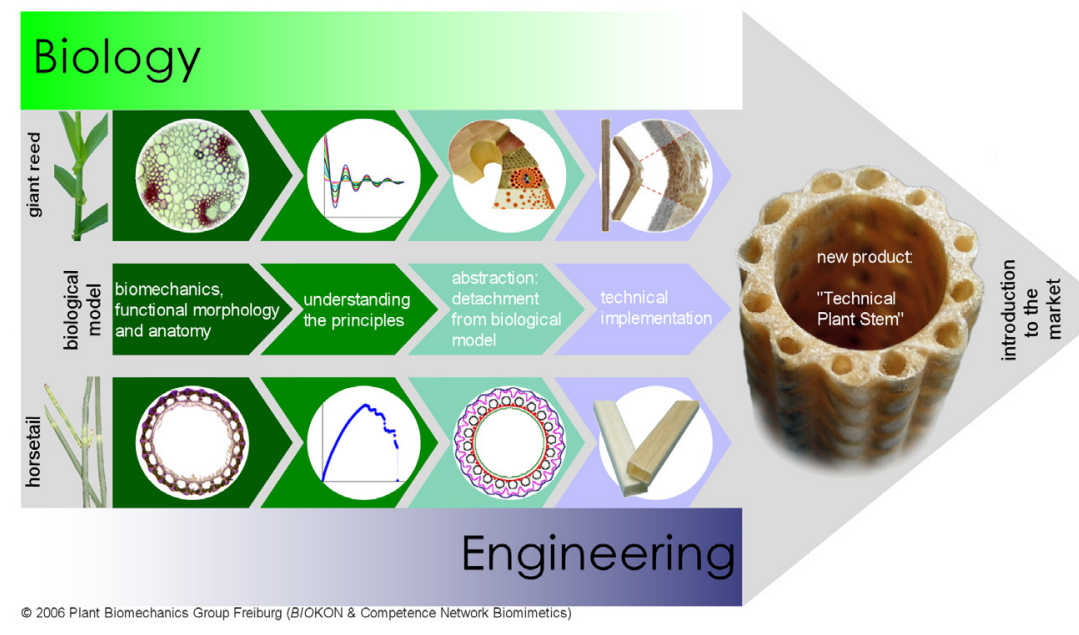

Figure 3: $\quad$ Bottom-up process: progression of a biomimetic project from the biological templates to the biomimetic product as exemplified by the 'technical plant stem' [4-6].

For a successful implementation in technical applications, biological insights have to be made understandable for non-experts in biology, i.e. the project partners from engineering, physics or chemistry. The next step deals with the technical implementation, which generally takes place first on a laboratory scale, and then on an engineering scale. In the latter, methods and production techniques are used that are already established in industry. Then the biomimetic products are optimised with respect to production sequences and costs. This step takes place in close cooperation with and often under the leadership of the 
industrial partners. Marketing by the industrial partner is then accompanied as required by flanking measures, e.g. advertising.

The entire process of biomimetics forms a continuum in the cooperation among biologists, engineers and other scientific and industrial partners. A biomimetic research project often passes through several iterative loops in order to reach satisfying (interim) results.

\section{Top-down process}

A biomimetic project following the top-down process typically starts with the work of an engineer. In this approach biomimetic innovations and improvements are sought for already existing technical products. These products might either be in a final state of industrial development, or are often already successfully established on the market, fig. 4. For a successful top-down process wellfounded expertise is required from company representatives (engineers) as well as from fundamental researchers (biologists), and also readiness to talk with the parties on both sides. The improvement or further development of an existing product stands in the centre of the cooperation during a top-down process.

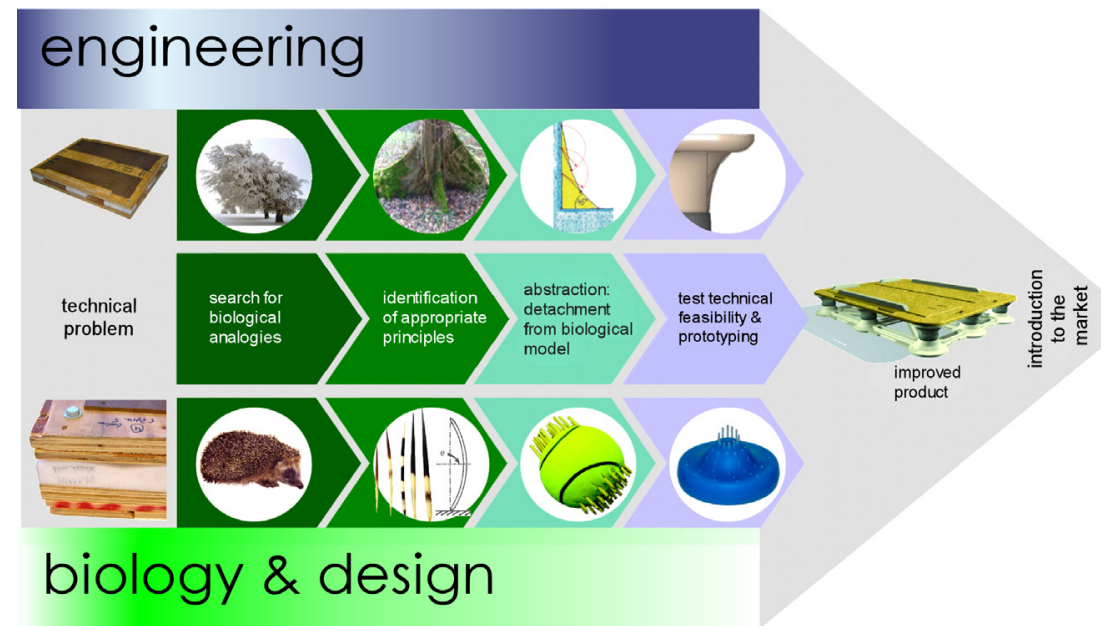

Figure 4: Top-down process: progression of a biomimetic research project from the biological templates to the biomimetic product as exemplified by the 'shock-absorbing transportation pallet' [7].

In the first step of a top-down process, the technical problem and its boundary conditions have to be precisely defined. Then the biologist looks for natural examples of solutions that look promising to solve the technical problem. This screening process typically renders several to many biological templates which are combed through by biologists and engineers for the examples that fit best as concept generators for the specific technical requirements. 
One or two of the most promising biological solutions are then selected for further analysis and investigated experimentally and characterised as to their suitability for the problem.

As in the bottom-up process, the next step in the top-down process is the abstraction. This means separating the solutions found from their natural examples. The next step following a successful transfer of knowledge is the task of the engineer to investigate the potential for technical implementation. Subsequently, initial biomimetically optimised prototypes are produced and their performance is tested. If the tests are successful, the development is extended all the way to industrial production. Finally, the biomimetically improved product is introduced in the market by the industrial partner with flanking measures as discussed in the bottom-up.

\section{Extended top-down process}

In the case of the extended top-down process the progression of a biomimetic R\&D-project is very similar as described for the 'regular' top-down process. The extended version characteristically starts as a 'normal' top-down process, i.e. with the search for biomimetic innovations and improvements for an already existing technical product.

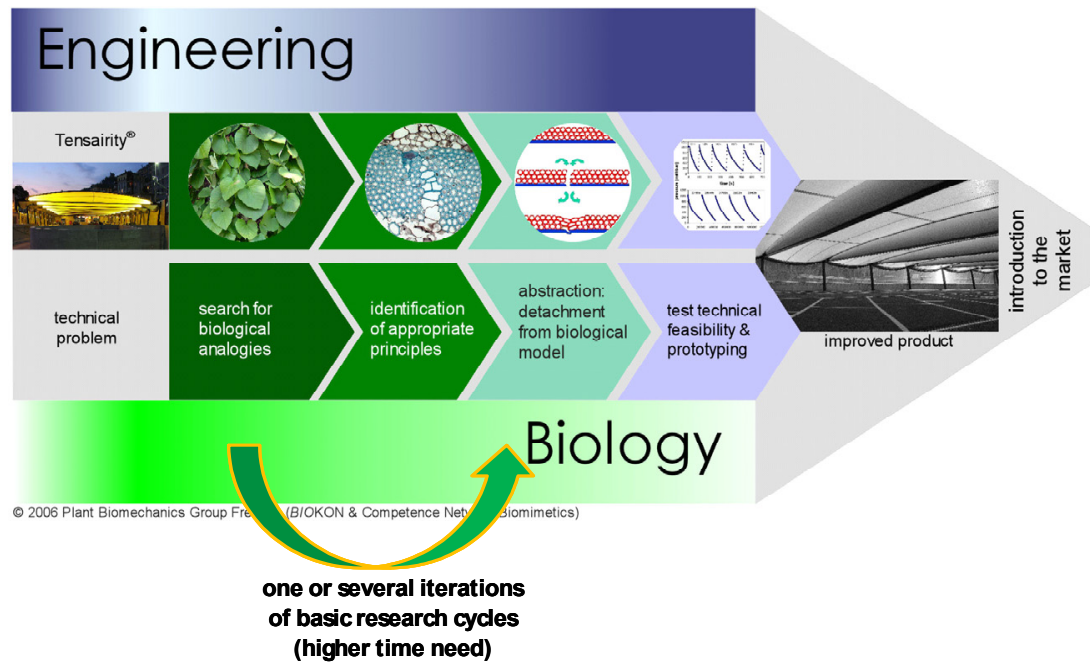

Figure 5: $\quad$ Extended top-down process: Progression of a biomimetic research project from the biological templates to the biomimetic product as exemplified by 'self-repairing membranes' for pneumatic Tensairity ${ }^{\circledR}$ structures [8-10].

In this case, the screening may offer some promising natural templates, but it also shows that there is a significant lack of knowledge i.e. of fundamental biological data. For this reason - sometimes after a successful first process cycle 
that may even result in first patentable results - it is decided, that there is need for more fundamental biological research. This insight leads to one or several iterations of the basic research cycles during an extended top-down process. Only by doing these cycles can it be guaranteed that the best or at least very good biological templates are used as concept generators for improving the technical product. Relaying on the limited database without the iteration cycles could result in a solution that renders a biomimetic improvement of the technical product which is limited due to the restricted existing biological knowledge. Further fundamental biological research with focus on the given technical question may help to find more suitable biological templates and serve to increase the biomimetic improvement considerably.

\section{Comparison of the three processes}

As discussed above for the development of biomimetic products, two fundamentally different methodologies can be distinguished according to their process sequences [1].

The starting point for a biomimetic development in the bottom-up process is the fundamental research of biologists. Being based on new insights in biological functions and structures, technical implementations developed during the bottom-up process, can often be applied to a large number of problems if the principle is understood and the abstraction has been successful. In this case the innovative leaps can be decidedly larger. However, a typical bottom-up process requires significantly longer time. Generally it takes several (three to seven) years, between the recognition of a biological function or structure interesting for technical implementation and the fabrication of an innovative biomimetic product resulting from it.

In the top-down process, on the other hand, an engineer wants to find out whether nature might have suggestions for solving his particular technical problems. Therefore he contacts a biomimetically working biologist. This approach can lead relatively quickly to the successful development of a biomimetically improved product. The time need in a top-down process typically ranges from six to eighteen months from posing the problem to the production of a functional demonstrator or a prototype. The limitation of the top-down process lies in the fact that the innovative leaps that can be expected are usually relatively small.

The extended top-down process is characterised by the insertion of several iterations of the basic research cycles after a first screening process. This is necessary to guarantee that a suitable biological template is used as concept generator. Sometimes, as in the case of the self-repairing membranes for pneumatic Tensairity ${ }^{\circledR}$ structures, after a successful first process cycle patentable results are found. However, as biology may have an even better solution as the one used in the first cycle, more fundamental research is needed. This approach may render great innovative leaps comparable to the ones found in the bottom-up process, but it is more restricted as to the range of application. And, also comparable to the bottom-up approach, it may also take several years from 
formulating the problem to the final biomimetic product. Typically the time need ranges between those of the top-down and bottom-up approach, i.e. between one and five years.

\section{Acknowledgements}

The authors thank all members of the Plant Biomechanics Group Freiburg for the successful collaboration in many biomimetic projects during the last decade. Our special thanks goes to our colleagues from the Institute for Textile and Process Technology (ITV) Denkendorf (Germany) and EMPA-Materials Science \& Technology Dübendorf (Switzerland). We also want to thank our industrial partners from Rittal $\mathrm{GmbH} \& \mathrm{Co} . \mathrm{KG}$, Herborn (Germany) and prospective concepts ag, Glattbrugg (Switzerland) for long-lasting and fruitful collaborations in several R\&D projects.

\section{References}

[1] Speck, T., Harder, D. \& Speck, O., Gradient materials and self-repair: learning technology from biology. VDI-Report, B 4284, pp. 1-13, 2007.

[2] Nachtigall, W., Bionik. 2. Aufl., Springer: Heidelberg, 2002.

[3] Nachtigall, W., Vorbild Natur. Bionik-Design für funktionelles Gestalten. Springer: Heidelberg, 1997.

[4] Milwich, M., Speck, T., Speck, O., Stegmaier, T. \& Planck, H., Biomimetics and technical textiles: solving engineering problems with the help of nature's wisdom. American Journal of Botany, 93(10), pp. 1295-1305, 2006.

[5] Milwich, M., Planck, H., Speck, T. \& Speck, O. The technical plant stem: a biomimetically inspired narrow fabric. Melliand - Narrow Fabric and Braiding Industry, 44(2), pp. 34-38, 2007.

[6] Rüggeberg, M., Burgert, I., \& Speck, T., Fibre-matrix interfaces in plants as model systems for technical composites. Proceedings of the 5th International Plant Biomechanics Conference Vol. I, Stockholm, ed. L. Salmen, STFI Packforsk AB: Stockholm, pp. 77-82, 2006.

[7] Masselter, T., Milwich, M., Monnerat, H., Scharf, U., Hartel, M. \& Speck, T., Bio-inspired solutions for technical problems: Biomimetic cable entries and shock-absorbing pallets, ed. C.A. Brebbia, Design and Nature IV, this volume, WIT Press: Southampton, 2008.

[8] Speck, T., Luchsinger, R., Busch, S., Rüggeberg, M. \& Speck, O., Selfhealing processes in nature and engineering: self-repairing biomimetic membranes for pneumatic structures, ed. C.A. Brebbia, Design and Nature III, WIT Press: Southampton, pp. 105-114, 2006.

[9] Speck, O., Luchsinger, R., Busch, S., Rüggeberg, M. \& Speck, T., Selfrepairing membranes for pneumatic structures: transferring nature's solutions into technical applications, Proceedings of the 5th International Plant Biomechanics Conference Vol. I, Stockholm, ed. L. Salmen, STFI Packforsk AB: Stockholm, pp. 115-120, 2006. 
[10] Busch, S., Speck, T., Liszkay, A., Speck, O. \& Luchsinger, R., Self-repair processes in plants as concept generators for innovative biomimetic technical materials with self-repairing functions. Proceedings of the 5th International Plant Biomechanics Conference Vol. I, Stockholm, ed. L. Salmen, STFI Packforsk AB: Stockholm, pp. 83-88, 2006. 